\title{
Théologiques
}

\section{La modernité, un défi pour le christianisme et l'islam}

\section{Claude Geffré}

Volume 9, numéro 2, automne 2001

Sens et spiritualité dans les pratiques professionnelles

URI : https://id.erudit.org/iderudit/007299ar

DOI : https://doi.org/10.7202/007299ar

Aller au sommaire du numéro

Éditeur(s)

Faculté de théologie de l'Université de Montréal

ISSN

1188-7109 (imprimé)

1492-1413 (numérique)

Découvrir la revue

Citer cet article

Geffré, C. (2001). La modernité, un défi pour le christianisme et l'islam.

Théologiques, 9(2), 135-156. https://doi.org/10.7202/007299ar d'utilisation que vous pouvez consulter en ligne.

https://apropos.erudit.org/fr/usagers/politique-dutilisation/ 


\section{La modernité, un défi pour le christianisme et l'islam ${ }^{1}$}

Claude GeFFrÉ, o.p.

Professeur émérite Institut catholique de Paris

Le thème que j'ai accepté de traiter devant vous est particulièrement ambitieux, surtout au lendemain des événements tragiques du 11 septembre 2001. Il est d'une telle complexité qu'il faudrait faire preuve d'une compétence beaucoup plus étendue quant à l'histoire passée et présente de l'islam pour être à la mesure du sujet. Mais, au moins en guise d'introduction, qu'il me soit permis de vous inviter tout de suite à dépasser certaines abstractions et certains stéréotypes qui sont inscrits dans notre mémoire collective.

En ce début du troisième millénaire, le christianisme et l'islam sont les deux premières religions du monde : le christianisme a déjà dépassé le milliard et demi de fidèles et l'islam le milliard. Mais de quel christianisme parle-t-on ? Au-delà des distinctions historiques entre catholicisme, protestantisme et orthodoxie, quelle différence y a-t-il entre le christianisme du premier monde (Europe et Amérique du Nord) et le christianisme des Églises dites du tiers-monde ? On constate de plus en plus que l'avenir démographique et spirituel du christianisme se joue moins en Occident que dans l'Amérique latine, l'Afrique et l'Asie. Le Vatican et le Conseil œcuménique des Églises en savent quelque chose et ne manquent pas de modifier leur stratégie.

Et de quel islam parle-t-on ? On peut difficilement dissocier l'islam de la civilisation arabo-musulmane, mais il faut se souvenir

1. Invité par la Faculté de théologie et le Centre d'étude des religions de l'Université de Montréal, Claude Geffré à prononcé à l'automne 2001 plusieurs conférences. Le texte qui suit développe les propos tenus au département de philosophie, sur le christianisme et l'islam face à la modernité. 
que l'islam asiatique est numériquement beaucoup plus important que l'islam des pays de langue arabe. Et quant à ces derniers, quelle différence y a-t-il entre l'islam du Maghreb et celui des pays du Golfe ? Il faut aussi prendre en compte la spécificité de l'islam de l'Afrique noire, et cette nouveauté de l'islam européen, avec déjà plus de 14 millions de fidèles.

En fonction de cette réalité plurielle, il ne faut pas simplement dépasser les abstractions. Il faut aussi purifier notre imaginaire. Cela vaut pour les chrétiens comme pour les musulmans. Alors que la modernité sous toutes ses formes est encore vécue comme une menace pour des religions comme l'islam et le christianisme lui-même, il serait tentant d'en rester à une menace réciproque de l'islam et du christianisme. Depuis l'effondrement du communisme, certains, comme l'auteur américain Fr. Fukuyama, sont prêts à décréter que la fin des idéologies coïncide déjà avec "la fin de l'histoire » et que nous sommes tous désormais sous le signe, à l'échelle mondiale, de l'idéologie néolibérale de la loi du libre-échange tous azimuts. C'est dans ce contexte de la prétendue "fin de l'histoire » que d'autres prophétisent déjà que le troisième millénaire sera de plus en plus caractérisé par le conflit des civilisations, et donc des grandes religions. On reconnaît là la thèse de Samuel Huntington dans son fameux article : « The Clash of Civilizations ${ }^{2} »$.

Mais dès que l'on compare l'islam et le christianisme, il reste vrai que nous n'évoquons pas seulement l'affrontement spirituel et doctrinal entre deux religions concurrentes. Dans nos mémoires collectives, il s'agit aussi de l'affrontement entre deux empires et deux civilisations. De même que le christianisme primitif a donné naissance à la chrétienté avec toutes les confusions possibles entre le politique et le religieux, le succès de la prédication du prophète a entraîné très vite l'édification d'un nouvel empire qui a conquis l'espace méditerranéen $\mathrm{du} \mathrm{VII}^{\mathrm{e}}$ siècle au XII ${ }^{\mathrm{e}}$ siècle, avec des capitales aussi prestigieuses que Damas, Bagdad et Cordoue. Et quoi qu'il en soit de la différence des situations historiques, encore aujourd'hui, l'affrontement entre les deux religions alimente la rivalité entre deux modèles de civilisation, le monde de l'islam d'un côté, l'Occident de l'autre. Et à l'intérieur de

2. S. Huntington, "The Clash of Civilizations ", Foreign Affairs, 72/3 (1993) p. 22-49. 
chaque religion, les fidèles projettent sur l'autre leur imaginaire collectif qui se nourrit de schèmes stéréotypés, de frustrations et de craintes ancestrales. La guerre du Golfe et le récent conflit des Balkans ont réactualisé l'antique peur de la chrétienté qui, jusqu'à la victoire de Lépante en 1571, a vécu la menace de l'islam avec la mentalité d'une ville assiégée. Et à cause des dérives récentes de l'islamisme, certains sont tentés de penser, surtout depuis la chute du mur de Berlin, que désormais la frontière entre le monde libre et un monde totalitaire est celle de l'Occident et du monde de l'islam ${ }^{3}$.

A l'inverse, l'imaginaire spontané des masses musulmanes continue souvent d'identifier le christianisme avec le modèle d'un Occident impérialiste et matérialiste. Et un discours simplificateur serait enclin à nous faire croire que le christianisme est la religion dominante des pays riches du premier monde, alors que l'islam serait la religion par excellence des pays pauvres du tiers-monde. On sait combien la réalité est autrement complexe, surtout si on évoque les pétro-dollars des pays du Golfe et les performances économiques de certains pays musulmans du Sud-est asiatique. Or, en fait, on constate que sur plusieurs continents, les Églises chrétiennes deviennent souvent les meilleures alliées des mouvements de libération des peuples opprimés, même s'il reste vrai que le christianisme doit s'interroger sur sa responsabilité historique directe ou indirecte dans l'édification d'un ordre mondial sous le signe de l'impérialisme de la loi du marché.

Cette introduction n'était pas inutile pour éviter d'imaginer une identité de situations du christianisme et de l'islam face au défi de la modernité. Il faut essayer maintenant d'avancer dans l'analyse du processus complexe de modernité. Je commencerai par évoquer le débat autour de la modernité dans la culture occidentale et les attitudes diverses des Églises par rapport à ce débat. Nous verrons ensuite comment se pose la question de la modernité à l'intérieur de l'islam. Nous nous demanderons enfin si sous le nom de mondialisation, la modernité n'est pas un défi commun que le christianisme et l'islam doivent relever ensemble en vertu d'une émulation réciproque.

3. J'avais déjà évoqué cette tentation néfaste dans "La responsabilité historique des trois monothéismes ", dans J. DORÉ, dir., Le Christianisme visà-vis des religions, Namur, Artel, 1997, p. 259. 


\section{La modernité en débat}

Modernité et postmodernité, les deux termes nous sont devenus familiers pour désigner un débat tout à fait typique du tournant d'un siècle à l'autre. Le débat concerne le destin même du christianisme dans le monde contemporain au moins en Occident. Son enjeu, en effet, c'est celui de l'avenir du christianisme dans des sociétés qui sont sous le signe à la fois de la modernité et de cette crise de la modernité dont la postmodernité est devenue l'indice. Le débat n'est pas seulement historique et sociologique, il est aussi théologique ${ }^{4}$. La question en effet est de savoir si la modernité, qui est toujours interprétée en termes de défi pour les religions en général, pourrait être considérée aussi comme une chance, au moins dans le cas du christianisme.

Selon une première approche de type sociologique, on peut désigner par modernité la nouvelle civilisation inaugurée à la fin du XVIII siècle par ces deux événements majeurs que furent la révolution industrielle et la révolution démocratique. Mais c'est en rester encore à une vision descriptive de la modernité. Si on veut déceler les racines de la dynamique propre au processus de modernisation, on doit faire appel aux concepts de subjectivité et de rationalité. Le passage à la modernité coïncide avec l'émergence d'un sujet humain conscient de son autonomie et avec la victoire d'une approche rationnelle de tous les phénomènes de la nature et de la société (qu'il s'agisse du progrès scientifique, de la mécanisation industrielle, de la rationalisation d'une économie de marché ou de la centralisation bureaucratique de l'État moderne). Or, sous la double modalité d'une subjectivité inviolable et d'une rationalité triomphante, on constate que le christianisme et la modernité entretiennent des rapports qui ne sont pas seulement faits de rivalité conflictuelle. On peut découvrir aussi des rapports de proximité.

\subsection{L'exclusion réciproque de la modernité et de l'Église}

Il est incontestable que, dans un premier temps, la version dominante des rapports du christianisme et de la modernité fut celle de leur exclusion réciproque. C'est surtout vrai du christianisme sous la

4. Je me permets de recommander le numéro La modernité en débat de Concilium, 244 (1992), dont j’ai rédigé l'éditorial. 
forme du catholicisme romain. Le catholicisme s'est voulu résolument anti-moderne dans la mesure où la raison des Lumières sapait l'autorité de la révélation et de la tradition et où l'avènement des sociétés démocratiques contestait directement le principe hiérarchique de la société-Église. À la suite du Syllabus de Pie IX contre les erreurs modernes, le catholicisme intransigeant fut la réponse historique qui voulait maintenir l'intégrité de la tradition chrétienne face aux prétentions sacrilèges de la modernité 5 .

Après la crise moderniste et après une longue période de tâtonnements ponctuée de tentatives régulières de restauration, il a fallu attendre Vatican II pour procéder à des discernements et comprendre que la sécularisation ne s'identifie pas nécessairement au sécularisme athée, que la liberté de conscience ne compromettait pas fatalement les droits d'une vérité objective, et que la séparation de l'Église et de l'État pouvait être la meilleure garantie d'une parole libre de la part de l'Église.

Il semble donc que le christianisme soit mûr pour une nouvelle négociation avec la modernité, alors que depuis deux siècles elles étaient sœurs ennemies, comme si la modernité était à sa racine antireligieuse et la religion essentiellement anti-moderne. En tout cas, en ce qui concerne le christianisme, certains historiens se demandent sérieusement si celui-ci ne fut pas lui-même un vecteur de la modernité alors même qu'il en fut finalement la victime. Ce serait le développement des virtualités propres au christianisme, en particulier l'émergence du sujet humain comme agent de l'histoire, qui serait à l'origine du monde moderne. Et quand ce phénomène de modernité a traduit toutes ses potentialités dans la vie des sociétés, il est devenu le rival toujours plus efficace du christianisme comme force historique. Ainsi, à l'époque moderne, le destin de la religion chrétienne, alors qu'elle n'est plus un facteur de cohésion sociale, peut être interprété comme celui de son effacement progressif inéluctable. On a reconnu la thèse de Marcel Gauchet, qui n'hésite pas à écrire que le christianisme est la religion « de la sortie de la religion ${ }^{6}$ ».

5. Parmi les très nombreux travaux d'É. POULAT consacrés au modernisme et au catholicisme intransigeant, citons au moins sa brève synthèse "Catholicisme et modernité. Un procès d'exclusion mutuelle ", Concilium, 244 (1992) p. 25-31. 
Cet auteur ne va pas évidemment jusqu'à dire que la fonction sociale du christianisme entraîne nécessairement la fin du christianisme comme expérience vécue et comme croyance individuelle. Mais on serait en droit de lui demander en vertu de quel postulat on doit décréter que l'essence du religieux se trouve à l'origine de l'humanité alors qu'il se définit encore par une totale soumission au divin et à l'ordre immuable de l'univers. Il semblerait que le religieux véritable ne s'épuise pas dans sa fonction de cohésion pour le groupe social. Si le christianisme a joué un rôle décisif dans l'accouchement de la modernité comprise comme avènement d'un sujet libre et autonome, ce n'est pas parce qu'il trahissait l'essence de la religion, mais bien plutôt parce qu'il inaugurait une désaliénation radicale de la religion, vécue enfin selon le registre de la libre réponse de l'homme au don de Dieu.

\subsection{Le phénomène de la post-modernité}

Jusqu'ici, j’ai parlé du défi de la modernité pour le christianisme dans le cadre classique en Occident d'une exclusion réciproque de la religion et de la modernité comprise comme processus de sécularisation de tous les registres de la vie humaine. Mais depuis déjà deux décennies, notre paysage culturel a profondément changé, et le vrai défi, c'est celui de la post-modernité. On peut à bon droit exprimer quelque réserve à l'égard de ce mot tellement flou de post-modernité 7 . Mais il n'est pas abusif pour désigner la contestation d'une modernité qui se définit par une raison trop sûre d'elle-même et nécessairement en conflit avec toute forme de religion. Cette post-modernité aura justement deux issues qu'il ne faut pas confondre : celle du retour du religieux et celle d'un nihilisme néo-païen.

Le destin de la sécularisation, c'est-à-dire du désenchantement du monde, est indissociable du processus de modernisation. Mais aujourd'hui nous ressentons le besoin de revoir les théories classiques de la sécularisation, dans la mesure où celle-ci n'est pas nécessaire-

6. M. GAUCHET, Le désenchantement du monde. Une histoire politique de la religion, Paris, Gallimard, 1985.

7. En dehors du livre déjà ancien mais symptomatique de J.F. LYOTARD, La condition postmoderne, Paris, Éditions de Minuit, 1979, il faut surtout se référer aux longues réflexions de J. HABERMAS, Le discours philosophique de la modernité, Paris, Gallimard, 1988. 
ment synonyme de déreligiosisation ${ }^{8}$. Avec le recul historique, nous portons un autre regard que le théologien Bonhoeffer sur la sécularisation et l'irréligion de l'homme moderne. Autre chose est d'affirmer la laïcité de nos sociétés, le dépérissement de beaucoup d'institutions chrétiennes, la perte de l'influence culturelle, politique et morale des Églises et d'en conclure que l'homme n'a plus le sens du sacré ou qu'il est devenu totalement irréligieux.

Comment concilier la sécularisation croissante de nos sociétés et ce phénomène dit du " retour du religieux » ? Certains l'interprètent évidemment comme le choc en retour d'une modernité qui n'a pas tenu ses promesses et qui, par excès de rationalisation et de planification, a conduit à un certain désenchantement du monde et de l'homme lui-même. Mais on peut comprendre aussi le retour du religieux comme l'expression de la modernité elle-même, si par modernité on n'entend pas seulement l'avènement de la raison critique, mais l'aspiration constante de l'homme à un plus-être. Il s'agirait de cette modernité psychologique que certains sociologues français désignent comme " haute modernité " ou encore "ultramodernité9 ». On a en effet dépassé le schéma banalisé selon lequel le religieux coïncide nécessairement avec une aliénation ou une projection illusoire. Il peut être aussi une modalité de la quête du bonheur. Comme le dit JeanPaul Willaime, "l'ultramodernité, ce n'est pas moins de religieux mais du religieux autrement ». Il semblerait que notre sensibilité contemporaine ait une plus vive conscience des limites de la rationalité moderne et soit en quête d'une rationalité émergente qui prenne davantage en compte les composantes affective, esthétique et éthique du « connaître » humain. On assisterait donc à un dépassement de l'opposition fatale entre la foi et la raison, cet héritage typique des Lumières. Selon l'avertissement du philosophe J. Habermas, il faut prendre en compte en tout cas la distance entre l'action communicationnelle (celle qui préside aux échanges humains), qui est l'œuvre de

8. J'emprunte volontiers ce mot à Danièle HERVIEU-LÉGER, qui y recourt de plus en plus, surtout dans son ouvrage Le pèlerin et le converti, Paris, Flammarion, 1999.

9. C'est ce dernier mot qu'adopte le sociologue Jean-Paul WiLLAIME pour désigner la modernité aboutie. Voir "Le christianisme, une religion de l'avenir de la religion ", dans R. RÉMOND, dir., Les grandes inventions $d u$ christianisme, Paris, Bayard, 1999, p. 241. 
la raison au sens le plus large (Vernunft) et l'action instrumentale, qui est l'œuvre de l'entendement technologique (Verstand).

L'étiquette commode de post-modernité peut donc servir à désigner ce large mouvement de réenchantement du monde et de l'homme, qui trouve son expression dans les diverses religiosités ou gnoses qui exercent une réelle séduction chez beaucoup de nos contemporains. Face à l'angoisse provoquée par le vide du sens et la fin des idéologies du progrès, de la science, du socialisme, il y a une aspiration générale à la fonction tutélaire du sacré qui jouerait comme le rôle d'une Mère originaire. Cela se retrouve dans les divers groupes qui relèvent du New Age ou encore de ce que l'on appelle " la nébuleuse ésotéro-mystique ", mais aussi dans les diverses mouvances de spiritualité extrême-orientale et même dans certains mouvements charismatiques au sein même des Églises chrétiennes ${ }^{10}$.

Mais le défi de la post-modernité pour le christianisme, ce n'est pas seulement l'explosion du religieux, c'est aussi le nihilisme de toute une part de la culture contemporaine, nihilisme qu'il ne faut pas confondre avec les humanismes athées ${ }^{11}$. Ce nihilisme est lié au succès des sciences humaines et à leur volonté anti-humaniste : il s'en prend aux illusions de la présence, du sujet dans sa volonté d'auto-fondation et d'une histoire linéaire porteuse de sens. Selon l'interprétation de Heidegger, on peut le considérer comme le point d'aboutissement de ce qui était inscrit en germe dans la métaphysique occidentale. Dieu, le fondement des étants, est devenu une idole conceptuelle. Mais si Dieu est mort, ce n'est pas pour que l'homme prenne sa place. Il faut compléter le récit de la mort de Dieu par la mort du sujet.

Ce nihilisme n'a pas nécessairement une issue sombre et désespérée. Il engendre plutôt dans certains milieux un néo-paganisme qui célèbre contre la tradition chrétienne toutes les promesses de la vie enfin retrouvée et qui se réclame volontiers de Nietzsche. Il y a désormais simplement la terre et le « jeu du monde ». Il s'agit de retrouver l'innocence du

10. Je renvoie à l'étude suggestive de J.L. SCHLEGEL, "Le 'réenchantement' du monde et la quête du sens de la vie dans les nouveaux mouvements religieux ", dans Y. TARDAN-MASQUELIER, dir., Les spiritualités au carrefour du monde moderne, Paris, Centurion, 1994, p. 85-101.

11. Voir à cet égard l'article très éclairant de D. TRACY, « La désignation du présent », Concilium, 227 (1990), p. 71-92. 
premier matin. Il y a en Occident tout un courant qui veut renouer audelà et contre la tradition judéo-chrétienne avec une sorte de paganisme antique et de polythéisme qui cherche à sacraliser le monde et la vie. Le temps linéaire inauguré par le messianisme biblique est une maladie de l'esprit qui a conduit aux totalitarismes modernes. Il faut célébrer la roue, le retour éternel de toutes choses, l'alternance du bonheur et du malheur. L'histoire n'a pas un unique sens. Elle est multiple, éclatée...

Au terme de ce large tour d'horizon de la modernité en débat, on serait tenté de faire une typologie des attitudes chrétiennes face à ce défi. Je n'ai plus le temps de le faire, mais on devine qu'il y a au moins trois types d'attitudes. Une attitude anti-moderne qui peut prendre la forme d'un néo-traditionalisme et même d'un intégrisme. Face aux effets pervers de la modernité, il s'agirait de revenir en deçà des acquis de la modernité comprise comme sécularisation, autonomie de la conscience, déliaison de la religion et de l'État. En second lieu, on peut discerner une certaine dérive libérale, celle des chrétiens qui cherchent à cumuler les ressources positives de la religion et de la modernité. Mais dans cette négociation, on peut se demander si le critère n'est pas trop uniquement le bien de l'homme « éclairé » ou "séculier » du monde moderne. Il y aurait une troisième voie, que j'appellerai volontiers prophétique et mystique, qui chercherait à réinterpréter le christianisme en fonction des complicités entre l'Évangile et les aspirations même de la modernité comme accomplissement toujours inachevé des possibles de l'homme.

\section{Les stratégies de l'islam face au défi de la modernité}

Il faudrait cumuler des connaissances historiques, sociologiques et géo-politiques pour tenir un discours pertinent sur le défi de la modernité pour l'islam. Et pourtant, la comparaison avec le christianisme est pleine d'enseignement quant à une meilleure intelligence du processus de modernisation dans ce qu'il a d'inéluctable.

\subsection{Remarques préalables}

Je commencerai par faire trois remarques préalables :

1) Il convient tout d'abord d'observer que le débat sur la post-modernité comme indice d'une crise de la modernité est un débat typiquement occidental et même européen. Au moment même où nous 
sommes plus critiques à l'égard d'un eurocentrisme longtemps dominant, il faut savoir que le monde s'européanise à grande vitesse, au moins au sens où la modernité technologique devient planétaire. Ainsi, les masses des pays en voie de développement et ce sont souvent des pays à majorité musulmane - aspirent à une modernité qui transformera leurs conditions de vie.

2) Le débat sur la modernité considérée du point de vue de l'islam est souvent biaisé, car la modernité est toujours associée à un Occident colonialiste, matérialiste et hédoniste. Même s'il y a une nécessaire modernisation de la vie quotidienne grâce aux produits de la civilisation technique, la réponse au défi de la modernité occidentale prendra la forme soit du fondamentalisme religieux, soit de mouvements proprement révolutionnaires. Depuis plus d'une décennie, plusieurs pays musulmans qui sont encore des pays pauvres cherchent une troisième voie au-delà de l'idéologie libérale des sociétés occidentales et du collectivisme des sociétés communistes. Cette troisième voie prendra la forme du retour à un islam pur et dur et à ses potentialités révolutionnaires. C'est le cas de la République islamiste d'Iran, dont la révolution impliquait que l'État se conforme à la guidance du chef religieux suprême. Mais c'est aussi l'idéologie des frères musulmans, qui sont actifs en Égypte et dans plusieurs pays du Maghreb et de l'Afrique noire.

3) Quand on compare le christianisme et l'islam face à la modernité, on ne doit jamais oublier le décalage historique entre les sociétés évoluées de l'Europe démocratique et la plupart des sociétés islamiques. Cela concerne tout à la fois une autre conception des rapports entre la religion et le pouvoir civil, une autre conception de l'autonomie des personnes et de leur liberté religieuse, une autre conception des rapports de l'homme et de la femme, un autre usage de la raison critique à l'égard des dogmes fondamentaux de la religion musulmane. Après un âge d'or à la fin du XII ${ }^{\mathrm{e}}$ siècle, la civilisation arabo-musulmane a connu une période de déclin et a accumulé les retards en comparaison avec l'évolution de l'Occident, surtout depuis le XVIII ${ }^{\mathrm{e}}$ siècle. Les historiens soulignent la décadence de l'empire ottoman tout au long du XIXe siècle. La disparition du sultanat et du califat a créé une situation toute nouvelle pour l'ensemble de l'islam. Le Réformisme musulman de la fin du XIX ${ }^{\mathrm{e}}$ siècle, sous la mouvance d'auteurs importants provenant surtout d'Égypte, fut une tentative extrêmement in- 
téressante d'adaptation des sociétés musulmanes à la modernité sans rien brader en matière de fidélité religieuse. Il s'agissait justement de pratiquer l'ijtihad comme effort personnel de recherche et d'interprétation en matière de science religieuse et juridique. Mais finalement, cette tentative a échoué. Elle fut jugée encore trop progressiste par les ulémas. Et on peut dire que le réformisme a cédé la place à l'islam politique, qui s'est inauguré avec la fondation de l'Association des frères musulmans par Hassan al-Banna, mort en 1946. Les frères musulmans luttent pour la création d'un État islamique, et leur projet fondamental, c'est l'islamisation de la société et de ses institutions sociales, juridiques et politiques. Leur combat est un jihad, une guerre sur la voie de Dieu ${ }^{12}$.

\subsection{Trois modalités de la modernité:}

Ces préalables étant posés, pour essayer de ne pas trop trahir la réalité complexe de l'islam dans sa réponse au défi de la modernité, je distinguerai volontiers trois modalités de cette dernière : la modernité scientifique et technique, la modernité politique et la modernité culturelle. Cela nous permettra de mieux apprécier à chaque fois une attitude qui est faite à la fois d'ouverture et de résistance.

1) Historiquement, la civilisation arabo-musulmane a contribué de manière éminente au développement des connaissances scientifiques. Encore aujourd'hui, on constate une grande ouverture aux résultats des découvertes scientifiques et une volonté de maîtriser toutes les technologies modernes. Au moins dans les pays musulmans les plus développés, il y a un grand attrait pour les produits les plus perfectionnés des industries de pointe, surtout dans le domaine des médias et de l'informatique. Et cette entrée dans la civilisation technique coexiste très bien avec un attachement inconditionné aux traditions religieuses et familiales les plus vénérables. C'est un peu comme si l'islam voulait donner la preuve que la révolution industrielle et technique n'entraînait pas fatalement une stérilisation du sentiment religieux et une sécularisation

12. Sur toute cette évolution particulièrement complexe, j'ai tiré grand profit des réflexions de Joseph MAILA dans "L'islam moderne entre le réformisme et l'islam politique ", dans F. LENOIR et Y.T. MASQUELIER, dir., Encyclopédie des religions, vol. 1, Paris, Bayard, 1999, p. 847-860. 
de la vie sociale, comme ce fut le cas en Occident. On pourrait d'ailleurs faire la même observation à propos de la nouvelle civilisation qui est en train de naître dans le sud-est asiatique. Mais il est permis d'avoir un jugement moins optimiste quant à l'avenir religieux de ces sociétés, surtout si on pense à la mentalité des plus jeunes. Il n'y a pas en effet d'usage neutre et innocent des produits de la technique moderne sans transformation profonde des modes de vie et des mentalités. Ils impliquent une rupture selon leur logique propre avec la conception mythique du monde. La sécularisation de la société et de la culture médiatique dominante entraîne nécessairement une certaine sécularisation de la conscience, au sens d'une transformation des structures de plausibilité de la religion.

2) La modernité politique est inséparable d'une modernité économique et sociale qui a transformé les rapports entre les hommes dans les sociétés occidentales. Il s'agit essentiellement de l'avènement d'un État de droit fondé sur le contrat social et non sur un principe transcendant, de la séparation de l'État et du pouvoir religieux, de la laïcité et de la reconnaissance du droit à la liberté religieuse. Sur ce terrain, il y a un déphasage complet entre les sociétés musulmanes et les démocraties occidentales. Je ne puis entrer dans les détails et faire état de régimes constitutionnels fort différents, soit qu'il s'agisse des États islamiques qui ont adopté la Charia comme loi à la fois religieuse et civile, soit de certains États modernes qui sont régis par une constitution civile qui protège théoriquement la liberté de culte de tous les citoyens. Les pays dont l'islam est la religion majoritaire ont accepté de signer la Charte des droits de l'homme de 1948 mais, sur le terrain, on constate souvent une interprétation restrictive du droit à la liberté religieuse ${ }^{13}$. En bref, on serait tenté de dire que l'attitude des autorités religieuses officielles de l'islam à l'égard des libertés modernes est assez proche de l'attitude de l'Église catholique au moment du Syllabus de Pie IX, en 1864. L'islam reproche à l'Occident en général d'avoir exalté les droits de l'homme au détriment des droits de Dieu. Comme le catholicisme intransigeant, il pense volontiers que la liberté

13. On lira avec les réserves qui s'imposent l'étude de R. GARAUDY, «Les droits de l'homme et l'islam » et celle de A.A. AN-NA'IM, "Qur'an, Shari'a et droits de l'homme " dans le numéro L'Éthique des grandes religions et les droits de l'homme de Concilium, 228 (1990). 
de conscience et la liberté religieuse favorisent l'athéisme. Il estime que les droits de la vérité révélée doivent toujours l'emporter sur les droits de la conscience individuelle.

Il y a là un débat fondamental sur la possibilité de concilier l'absolu de la vérité révélée et la modernité comprise comme raison critique et raison démocratique. L'Église catholique elle-même a mis des siècles à renoncer à l'idéologie de la vérité obligatoire et à proclamer à Vatican II que « la vérité ne peut s'imposer que par la force propre de la vérité » (cf. la Déclaration sur la liberté religieuse), c'est-à-dire sans contrainte directe ou indirecte. L'islam n'en est pas encore là. Il y a un lien étroit entre le droit à la liberté religieuse et la laïcité, et l'enjeu de la laïcité c'est celui-là même de la démocratie. Mais pour que les musulmans acceptent une certaine laïcité qui sera de plus en plus l'exigence même de toute société moderne comme société démocratique et religieusement pluraliste, encore faut-il ne pas en rester à une laïcité qui soit une laïcité anti-religieuse. Cette nouvelle laïcité comme vivre ensemble des citoyens invite les autorités religieuses à renoncer à toute forme d'hégémonie directe ou indirecte sur la société civile, mais elle invite aussi l'État à renoncer à une laïcité militante qui était une quasi-religion dans la France des droits de l'homme ${ }^{14}$. On peut espérer que l'islam européen qui se développe sous nos yeux favorisera l'émergence d'un certain type de citoyen musulman qui apprend à concilier son appartenance à l'umma musulmane et son appartenance à la société civile dans le respect de sa légitime autonomie.

3) Quand j'essaie d'apprécier l'attitude la plus commune de l'islam face à la modernité culturelle, je ne désigne pas par là le dernier état de la culture occidentale sous le signe de la post-modernité. Je ne pense pas non plus à la culture consumériste et hédoniste mondialement diffusée par les médias. Je vise un certain régime moderne de l'esprit sous le signe de la raison critique et de la conscience historique. Les intellectuels musulmans ont généralement adopté les méthodes et les procédures qui sont communes à toute recherche dans le domaine des sciences exactes et des sciences humaines. Mais

14. Parmi beaucoup de travaux en ce sens, je renvoie à J.P. WILLAIME, « La laïcité à la française ", Lumière et Vie, 188 (1990) et à l'étude de R. RÉMOND, "La laïcité ", dans Les grandes inventions du christianisme, Paris, Bayard, 1999, p. 97-107. 
si on envisage plus précisément l'application de la méthode historico-critique et des diverses ressources de la linguistique moderne à l'étude des textes fondateurs de l'islam, alors il faut bien reconnaître qu'il y a un fossé entre l'islam et le christianisme du point de vue de l'état des sciences religieuses. Ce qui est devenu un bien commun des exégètes et des théologiens chrétiens depuis près de cent ans demeure encore suspect dans la théologie officielle de l'islam.

Cette différence entre le christianisme et l'islam dans l'approche des Écritures fondatrices ne tient pas seulement au déphasage historique entre la culture occidentale et la culture dominante du monde arabomusulman que j'ai déjà évoqué ; elle ne provient pas non plus uniquement du fossé entre les Écoles coraniques et l'enseignement supérieur des universités, du point de vue de l'ouverture aux exigences modernes du savoir. Cette différence a des racines proprement théologiques. En dépit de certaines analogies, chrétiens et musulmans n'ont pas la même conception de la révélation et de l'inspiration quand ils essaient de réfléchir sur la distance entre l'Écriture et la Parole de Dieu ${ }^{15}$.

Il y a bien sûr toute une tradition herméneutique dans l'histoire de la pensée musulmane. Mais l'instance herméneutique au sens moderne du mot n'est pas vraiment prise au sérieux quand il s'agit d'entreprendre une relecture contemporaine du Coran ou des hadith du Prophète. Il faut se souvenir en effet que pour l'orthodoxie musulmane, Mohammed n'est pas l'auteur du Coran, même pas au sens où la théologie chrétienne parle d'un auteur inspiré qui écrit sous la mouvance de l'esprit divin. Il n'est qu'un transmetteur passif. C'est Dieu lui-même qui est l'auteur et Mohammed est désigné comme le sceau de la prophétie parce que le Coran qui lui a été dicté constitue le livre parfait, inimitable, qui non seulement achève la Révélation, mais fournit le critère à partir duquel on peut discerner ce qui est authentique et ce qui est falsifié dans les Écritures antérieures juives et chrétiennes. Il est donc insuffisant de dire que le Coran est inspiré à la manière où la foi chrétienne considère la Bible comme un livre inspiré.

15. J'ai tenté une étude comparative entre révélation chrétienne et révélation coranique à l'occasion des travaux de Mohammed Arkoun: "Révélation chrétienne et révélation coranique. À propos de la raison islamique selon Mohammed Arkoun " Revue des sciences philosophiques et théologiques, 81 (1997) p. 239-252. 
Aux yeux des musulmans, l'Évangile par exemple est déjà falsifié parce qu'à l'exception de quelques paroles de Jésus, il assume en fait la tradition interprétative de la première communauté chrétienne sur Jésus. Dans la perspective musulmane, l'idée de tradition interprétative n'a pas de sens puisque le Coran tout entier a été directement dicté par Dieu au Prophète. Et même si l'établissement du Coran comme corpus canonique a duré plus de vingt ans après la mort du Prophète, cette mise par écrit des sourates était la pure retranscription de l'enseignement direct du Prophète. Ainsi, l'idée d'une instance herméneutique qui ne compromette pas le caractère sacré de la Parole de Dieu est impensable dans le cas de l'islam. Même si plusieurs intellectuels musulmans commencent déjà à mettre le texte du Coran à l'épreuve de la critique historique et de la critique littéraire, je constate que leur liberté herméneutique est en conflit avec le dogme de l'infaillibilité de l'écriture coranique comme Parole de Dieu ${ }^{16}$.

\section{Le défi commun de la mondialisation pour l'islam et le christianisme}

J'ai donc insisté jusqu'ici sur tout ce qui différencie l'islam et le christianisme dans leur réponse au défi de la modernité. Ce n'est pas seulement la rivalité entre deux grandes religions à vocation universelle. C'est aussi la rivalité entre deux grandes civilisations. Mais en ce début du troisième millénaire, l'une et l'autre religion doivent affronter le phénomène moderne de la mondialisation. Et face à ce destin commun, il est permis de penser que leur rivalité ancestrale pourrait prendre la forme d'une émulation réciproque dans la conscience d'une responsabilité historique commune au service de la communauté mondiale. Les événements tragiques du 11 septembre 2001 ne sauraient compromettre cette chance historique. Ils pourraient au contraire inviter l'islam à affirmer son identité véritable et à prendre ses distances vis-à-vis des dérives aberrantes de l'islamisme.

16. On peut se reporter à l'ouvrage collectif du GRIC (Groupe de Recherches Islamo-Chrétien), Ces Écritures qui nous questionnent. La Bible et le Coran, Paris, Le Centurion, 1987. Mais les intellectuels musulmans qui s'expriment dans ce livre ne prétendent pas représenter le point de vue de l'islam officiel. 
Il est devenu banal de souligner l'ambiguïté du phénomène de mondialisation qui coïncide avec l'âge planétaire de l'humanité. Selon une première approche, on insistera plutôt sur les effets bénéfiques de cette globalisation qui a favorisé l'extension à l'ensemble de la planète d'une certaine rationalité scientifique et technique née en Occident et qui s'est soldée par un progrès incontestable pour des millions d'êtres humains qui étaient encore soumis aux fatalités aveugles de la nature. Elle a aussi mis en valeur l'unité de l'esprit humain au-delà de l'éclatement des particularismes ethniques et culturels et elle a donc hâté l'émergence d'une éthique globale. Mais dans le monde tel qu'il est et par suite d'un réseau de communication toujours plus performant, c'est l'impérialisme de la loi du marché qui est le plus souvent le moteur caché du phénomène de mondialisation. Notre "village planétaire » tend à devenir un marché mondial, et le mimétisme de la consommation renforcé par le pouvoir fantastique des médias peut donner l'illusion d'une réelle solidarité entre les hommes. Ainsi, le système qui se met en place un peu partout est plutôt générateur de misère pour les trois quarts de l'humanité - deux milliards d'êtres humains vivent avec moins de deux euros par jour !,sans parler de la dégradation de l'environnement.

Le double écueil de la mondialisation, c'est donc tout à la fois un processus de globalisation qui tend à sacrifier les identités culturelles et religieuses, et par réaction, un phénomène de fragmentation qui conduit aux crispations identitaires et aux rivalités violentes pour la conquête du pouvoir économique et politique.

Face au double écueil de cette modernité inéluctable qu'est la mondialisation, je pense que le dialogue de ces deux grandes religions mondiales que sont le christianisme et l'islam est d'un enjeu considérable pour l'avenir d'une civilisation planétaire. Je me contenterai de quelques remarques. Je distinguerai le respect de l'humain authentique, une vocation prophétique contre l'injustice structurelle du monde et le sens d'une justice écologique.

\subsection{Le respect de l'bumain authentique}

J'ai longuement évoqué la rivalité historique entre l'Occident et la civilisation arabo-musulmane. Mais quand on évoque les racines spirituelles de l'Europe, en particulier la longue tradition judéo-chrétienne, on ne peut occulter sa composante méditerranéenne et 
l'importance de la tradition prophétique de l'islam. On peut parler à la suite de Mohammed Talbi d'un humanisme islamo-judéo-chrétien qui est d'un grand prix pour l'ensemble de la civilisation mondiale. À l'âge de la fin de l'eurocentrisme, nous devons dépasser notre mauvaise conscience post-coloniale et découvrir le prix d'un certain esprit européen pour lutter contre les effets déshumanisants d'une certaine culture mondiale véhiculée par les médias, une culture consumériste et hédoniste qui met en danger les identités culturelles. Le christianisme et l'islam peuvent être des instances de sagesse pour maintenir une certaine qualité de l'humain authentique ${ }^{17}$. Le génie de l'Europe est au point de rencontre de la tradition biblique et de la raison critique qui est un héritage de la Grèce et de l'âge des Lumières. Or tout au long de la conquête coloniale, les occidentaux n'ont exporté qu'un esprit européen tronqué, à savoir un appétit de domination, la maîtrise technique de la nature et le mépris des cultures locales.

Une rencontre cordiale avec l'islam pourrait nous aider à retrouver le sens de l'humain authentique, c'est-à-dire un homme qui ne se définit pas seulement en termes de besoins et d'échanges dans le sens de ses utilités immédiates. Il se définit en termes de désir et de dépassement de son désir. Disons le mot : il se définit aussi par l'ouverture à une Altérité transcendante. La meilleure preuve, c'est que le processus de sécularisation inhérent à la modernité ne réussit pas à écarter la revanche périodique de l'irrationnel. C'est comme si l'emprise croissante du technico-économique dans les sociétés contemporaines avait libéré un imaginaire déréglé qui favorise l'émergence, soit d'un sacré sauvage, soit d'un nihilisme néopaïen, soit encore le repliement sur un individualisme jaloux qui donne une importance démesurée à l'argent, au sexe et à la réussite sociale.

Par contraste avec le sacré archaïque ou sauvage du néopaganisme, le vrai sacré coïncide avec l'authenticité de l'humain véritable. Il y a en effet une complicité entre le sacré de la foi monothéiste qui est plus d'ordre éthique que sacrificiel et le sacré humain non religieux. L'islam et le christianisme n'ont pas le monopole du sacré, mais avec d'autres instances d'ordre éthique et culturel, ils ont pour vocation de

17. Je m'explique sur ce que j'entends par humain authentique dans mon livre Profession théologien. Quelle pensée chrétienne pour le XXI siècle?, Entretiens avec Gw. Jarczyk, Paris, Albin Michel, 1999. 
maintenir le sens de l'homme comme « histoire sacrée ». Face au nihilisme moderne, mais face aussi à la séduction des sagesses immanentistes de l'Orient, le christianisme et l'islam doivent faire la preuve qu'il n'y a pas de contradiction fatale entre la rencontre d'un Dieu personnel et le respect de l'humain authentique. La relation authentique avec Dieu peut être au contraire à l'origine d'un surcroît d'humanité pour soi-même et pour l'ouverture à autrui.

\subsection{La vocation prophétique des deux monothéismes}

Le christianisme et l'islam sont des religions qui annoncent un salut éternel de l'homme. Elles témoignent donc d'une espérance au-delà des limites de cette histoire et font ainsi la preuve qu'elles ne s'épuisent pas dans le service du monde. Mais en même temps, sur la base même de la révélation biblique ou du Coran, elles ont une dimension éthique et prophétique qui contribue à donner un visage humain à l'histoire. C'est évident dans le cas du christianisme qui, à l'exemple de Jésus, prolonge le dynamisme messianique des grands prophètes du premier Testament. Mais c'est vrai aussi de plus en plus de certains courants à l'intérieur de l'islam, qui font preuve d'une action proprement révolutionnaire contre les diverses aliénations dont des millions d'hommes et de femmes sont les victimes. On pourrait les apparenter aux théologies de la libération à l'intérieur du christianisme. Je me garde bien évidemment de confondre ces courants avec l'islamisme de Ben Laden, cet islamisme terroriste qui est beaucoup plus soucieux de déstabiliser l'Occident que de porter remède aux fractures et injustices du monde contemporain. C'est la chance du dialogue interreligieux de favoriser une prise de conscience commune quant à la responsabilité historique de chaque religion à l'égard de l'avenir de l'homme et de la création elle- même ${ }^{18}$. Concrètement, le christianisme et l'islam ont mieux compris qu'ils ne peuvent justifier leur prétention à l'universel que s'ils épousent les causes universelles de l'homme contemporain : le combat pour la justice, la défense et la promotion des droits de l'homme, la sauvegarde de la création, le respect de la vie, le souci prioritaire des plus défavorisés.

À l'âge de la mondialisation, nous ressentons le besoin d'une éthique globale qui bénéficie à la fois des ressources morales des grandes

18. Voir C. GEFFRÉ, " Maîtrise, chaos, salut », Le Supplément, 204 (1998) p. 177-195. 
traditions religieuses et de l'apport des éthiques séculières. Nous sommes en effet parvenus à un certain consensus éthique en matière de droits de la personne humaine qui a trouvé son expression officielle dans la Charte des droits de l'homme. Le christianisme et l'islam doivent donc se laisser interroger par la nouvelle conscience des aspirations légitimes de la personne humaine. Mais, à leur tour, les religions monothéistes ont une vocation prophétique à l'égard d'une simple éthique des droits de l'homme ou à l'égard d'une sorte de religion de l'humanitaire qui prétendrait avoir pris la relève des religions historiques. Certes, il n'est pas nécessaire d'appartenir à une religion pour lutter pour la défense et la promotion des droits de l'homme. Le fondement immédiat des droits de l'homme, c'est un certain consensus relatif à la dignité inviolable de toute personne humaine. Mais la violation permanente des droits de l'homme dans le monde contemporain tendrait à démontrer que la Charte des droits a besoin d'un fondement plus radical. L'expérience du cruel $\mathrm{XX}^{\mathrm{e}}$ siècle qui vient de s'achever nous a montré la fragilité de la conscience humaine laissée à elle-même. Même si, comme on l'a vu, l'islam parvient encore mal à concilier les droits de l'homme et les droits de Dieu, il peut nous aider à maintenir une distance critique à l'égard d'un discours quelque peu incantatoire sur les droits de l'homme.

Le fondement radical de la dignité inviolable de la personne humaine est posé avec la révélation biblique sur la création de l'homme à l'image de Dieu. C'est là un héritage commun à la Bible et au Coran, même si l'expression "image de Dieu " ne se trouve pas littéralement dans la révélation coranique. On y trouve en tout cas l'idée que l'homme est le calife de Dieu sur la création. Cela veut dire concrètement qu'il est impossible de porter atteinte aux droits de l'homme sans s'attaquer à Dieu même, et donc sans commettre un véritable blasphème. C'est parce que l'homme est l'« image de Dieu » que la vie de tout être humain, aussi négligeable soit-il, revêt un prix sacré. La création de l'homme à l'image de Dieu fonde l'égalité de tous les hommes entre eux. Et dans l'Ancien Testament, le droit de Dieu est au service des droits de l'homme. À cet égard, on ne saurait trop souligner l'importance du thème de la " justice de Dieu ». Mépriser les pauvres, les opprimés, les affligés, les étrangers, c'est porter atteinte au droit de Dieu ${ }^{19}$.

Ainsi, même si ni le christianisme ni l'islam n'ont la prétention de constituer une alternative sociale et politique pour rendre la terre plus habitable et la communauté des hommes plus conviviale, ces deux 
grandes traditions religieuses peuvent exercer un rôle de contre-culture à l'égard d'une culture dominante à l'échelle planétaire qui risque d'abîmer l'homme. Et, en même temps, elles ont vocation pour adresser un avertissement prophétique face aux injustices structurelles d'un monde qui obéit de plus en plus à la seule loi du profit et qui donc creuse toujours davantage le fossé entre nations riches et nations pauvres.

\subsection{La justice écologique}

La chance historique d'un nouveau dialogue entre l'islam et le christianisme, ce n'est pas seulement de rivaliser pour l'avènement d'une communauté humaine mondiale plus juste. C'est aussi de prendre en charge le destin même de la Planète Terre. Pour la première fois, nous découvrons qu'il ne suffit pas de défendre les droits de l'homme si nous ne respectons pas en même temps les droits de la Terre, pour reprendre l'expression mise en avant par Michel Serres. Certains parlent déjà de ce que pourrait être une justice écologique. Les pouvoirs de la science et des technologies modernes sont tels en effet que nous pouvons commettre des crimes contre l'identité du génome humain et contre les équilibres qui assurent la permanence de la vie sur la terre. La question-clé pour l'avenir, c'est la nature de la maîtrise rationnelle de l'homme. Comment prévenir les effets pervers de ce que nous expérimentons aujourd'hui comme un progrès ? Comment faire en sorte que la terre soit encore habitable pour les générations qui nous suivent. On pourrait rappeler ici le nouvel impératif catégorique mis en avant par Hans Jonas dans son Principe de responsabilité : "Agis de telle sorte que les effets de ton action soient compatibles avec la permanence d'une vie authentiquement humaine sur la terre ${ }^{20}$ ". C'est toute la question de l'autolimitation du pouvoir humain.

Face à cette éventualité d'une catastrophe écologique d'ordre planétaire, nous ressentons l'urgence d'une théologie de la création qui donne un fondement radical à notre confiance dans l'avenir, dans la

19. Pour l'enquête biblique sur les fondements scripturaires des droits humains, on se reportera utilement à R. COSTE, Les dimensions sociales de la foi. Pour une théologie sociale (Cogitatio Fidei 217), Paris, Cerf, 2000, surtout le chapitre 1.

20. H. JONAS, Le principe responsabilité. Une éthique pour la civilisation technologique / trad. par J. Greich, Paris, Editions du Cerf, 1990 (allemand 1979). 
vie, dans l'être. L'énigme de l'histoire demeure entière. Mais en vertu de la foi au Dieu créateur, chrétiens et musulmans savent que le dessein de Dieu, c'est la réussite de la création et l'accomplissement de la vocation de l'homme, image de Dieu comme intendant du monde qui lui a été confié. En dépit de tous les démentis, nous ne connaissons pas toutes les ressources des libertés humaines quand elles se mobilisent pour inverser le cours fatal de la violence et réaliser tous les possibles de l'histoire. Nous savons que la vocation de l'être humain est d'être un cocréateur avec Dieu en vue de rendre la terre habitable. Mais la transformation de la nature et l'exploitation des ressources de la planète ne doivent pas conduire à une démesure prométhéenne. Il semble que la pensée chrétienne aurait tout intérêt à retenir quelque chose du sens admirable de la beauté et de la stabilité du monde créé dont témoigne le Coran. En tout cas, nous portons déjà un regard plus critique sur certains théologiens chrétiens qui, sous l'influence de l'idéologie moderne du progrès, ont été tentés de réinterpréter les premiers chapitres de la Genèse dans un sens très anthropocentrique.

Finalement, face aux menaces qui pèsent sur l'environnement de l'homme dans un avenir plus ou moins proche, nous devons faire l'apprentissage d'une certaine démaîtrise. C'est le seul moyen d'endiguer une volonté de domination qui s'enivre de son propre pouvoir et qui ne respecte plus les équilibres fondamentaux qui peuvent assurer la survie de l'espèce humaine. Nous sommes en dette vis-à-vis des générations à venir pour que la terre soit encore habitable. Dans la fidélité au meilleur de la tradition biblique, les deux grandes religions directement confrontées à la modernité sous le signe des prouesses techniques doivent être des instances de sagesse. De même que Dieu s'est reposé le septième jour, l'homme du troisième millénaire doit découvrir le secret d'une sagesse sabbatique, c'est-à-dire, au-delà du vertige d'une activité démiurgique, le prix de la non-action, du silence, de la louange et de l'émerveillement devant la création.

\section{RÉSUMÉ}

Quel rapport existe-il entre les deux principales religions du monde, le christianisme et l'islam, et la modernité ? Après avoir signalé la complexité des réalités chrétienne et musulmane, et dénoncé les préjugés qu'entretiennent l'une envers l'autre les deux religions, l'auteur montre comment se pose la question de la modernité à l'intérieur de chacune d'elles. Si cette comparaison met en évidence des différences certaines dans la réponse de chacune au défi que représente la modernité, elle n'est pas sans 
révéler des solidarités au plan des enjeux : respect de l'humain authentique et respect de son environnement, tous deux inscrits au fondement même de ces religions.

\section{ABSTRACT}

This paper evokes the debate around modernity in Western Culture, through clarifying the relations between religion, modernity and postmodernity, and it examines the Christian's diverse positions concerning that debate. Next it studies the Islam's attitude facing modernity in its varied modalities, scientific and technical, politic, cultural. Among many differences brought up between them, in spite of some analogies, we notice that Christians and Muslims do not have the same conception of the revelation and the inspiration, especially when they try to think about the hermeneutic distance between the Scripture and God's Word. Hermeneutic freedom is in conflict with the dogma of infallibility of the koranic scripture as God's Word. We are wondering if, in the present historical situation, globalization can become a common challenge that Christianity and Islam, these ancient adversaries, could take up together, in accordance to reciprocal emulation. 